Research Article

\title{
Numerical Study on Different Series Modes of Jet Fan in a Longitudinal Tunnel Ventilation System
}

\author{
Guihong Pei and Jie Pan \\ School of Civil Engineering and Architecture, Southwest Petroleum University, Chengdu 610500, China \\ Correspondence should be addressed to Jie Pan; 1120400231@qq.com
}

Received 26 May 2014; Revised 21 August 2014; Accepted 4 September 2014; Published 29 September 2014

Academic Editor: Jaromir Horacek

Copyright ( 2014 G. Pei and J. Pan. This is an open access article distributed under the Creative Commons Attribution License, which permits unrestricted use, distribution, and reproduction in any medium, provided the original work is properly cited.

\begin{abstract}
Efficient ventilation systems will contribute to maintaining air quality in the tunnel. In order to improve ventilation performance in normal traffic condition, the 3D tunnel models were established according to the original design for the tunnel located in central plains of China. Based on the commercial CFD software Fluent 6.3 and $k-\varepsilon$ turbulence model, numerical simulations were carried out to study the patterns of jet flow and the optimization of fan combinations. It is found that the axial velocity profile obtained from numerical simulation agrees quite well with turbulent free jet theory although there is a little difference on the magnitude. The comparison of four combination modes under the condition of operating four fans indicates that the ventilation effectiveness is affected mainly by both the interval of adjacent groups of fans and the combination modes of operational fans. According to the simulation results, a novel combination mode which consists of a group double paralleled fans and two groups single fan is designed. The novel combination mode is regarded as the optimum combination mode with respect to maximizing air velocity in the tunnel. Compared to the traditional combination modes, it will increase the air velocity by $5.7 \%$.
\end{abstract}

\section{Introduction}

The air quality inside tunnel is gradually taken seriously because of the increasing of tunnel length. Owing to the special structure of tunnels, when a fire occurs, the smoke spreads fast and widely, which prohibits the safe evacuation of occupants and the firefighters from extinguishing the fire, while, in most tunnels, the ventilation system plays a crucial role in controlling the smoke and maintaining acceptable conditions within the tunnel during evacuation, rescue, and firefighting procedures [1]. Statistics have shown that smoke is the most fatal factor in fire and about $85 \%$ of victims in building fire were killed by hot and toxic smoke [2]. Therefore, efficient ventilation in a road tunnel is extremely important to prevent the smoke and the harmful substances from affecting tunnel users. The longitudinal ventilation mode with jet fans is highly anticipated due to its flexibility, reliability, lower cost, and easy control. Therefore, it not only gets the favor of engineers to be used in most of the tunnel but also causes the attention of scholars to do deeper research.

The four main methods of studying tunnel ventilation at home and abroad are theoretical calculations, model tests, field measurements, and numerical simulations. For example, Ohashi et al. used numerical method to study the vertical concentration distribution of pollutants in Kan-Etsu highway tunnel of Japan which is $10.885 \mathrm{~km}$ long. The results had provided the basis for design of ventilation and air pollution control in highway tunnel [3]. Rohne carried out an experimental research in which the fans were located near a flat plate to study the resistance and jet thrust. The influence of different installation separation of fans on the ratio of resistance and thrust had been investigated; at the same time, the influence of various distances from the plate on the ratio of resistance and thrust also had been analyzed [4]. Gao of Lanzhou Jiaotong University had analyzed the characteristics of jet flow and the pressure-rise of jet fan. Not only the relationship between the pressure-rise and the induced length but also the relationship between the pressure-rise and the separation of jet fans had been investigated by him [5]. Zhong of South China University of Technology had conducted field measurements in Dabaoshan tunnel, and the test results provided valuable data for the installation location of jet fans in longitudinal ventilation system [6]. According to the ideas of the composite structure method, Wang and Xia of 
Chang'an University had carried out numerical simulations on longitudinal ventilation, and the optimization ventilation method was picked out [7]. Colella et al. [1, 8, 9] had conducted a large number of researches in the field of tunnel ventilation, and they had obtained plenty of important results. They applied multiscale modeling approach to study the ventilation flows and fire in a tunnel domain. The multiscale modeling approach uses different levels of details when describing the fluid flow in different tunnel regions. The far field region is modeled by using a 1D "network" model and the near field region is modeled by using a $3 \mathrm{D}$ CFD model. The "network" and the CFD models exchange information at the 1D-3D interfaces [8]. The adoption of multiscale modeling approach allows for a more rational use of the computational resources without loss of accuracy [9]. The research results obtained by scholars mentioned above had laid the foundation for the study of tunnel ventilation system. All the scholars had put forward some valuable opinions.

The longitudinal ventilation systems with jet fans have been continuously updated. On the one hand, as the width of the tunnel increases, 2 or 3 jet fans are paralleled and suspended in the tunnel vault. On the other hand, the number of jet fans in series increases with the increasing of the tunnel length. In general, only the total number of operational jet fans will be given in design plan, while the combination mode of jet fans in normal traffic condition is not stipulated. The wind speed is different in various combination modes, and so it is the air pressure. Therefore, there is clear need to analyze the difference of pressure distribution and velocity distribution in various combination modes. Numerical simulations were carried out to study the air flow and the optimization of fan combinations inside tunnel based on CFD software Fluent 6.3. According to the simulation results, the best combination mode which is conductive in improving the ventilation performance in the tunnel is selected.

\section{The Mathematical Model}

The jet flow of fans in the tunnel can be described as follows: the velocity of gas has increased to $20 \sim 30 \mathrm{~m} / \mathrm{s}$ when flowing through the jet fans. Then, the jet flow sprays into the tunnel where the velocity of surrounding gas is about $3 \mathrm{~m} / \mathrm{s}$. Because of the entrainment between jet flow gas and surrounding gas, the vortex is formed which leads to the lateral pulse. Then, the jet flow has momentum exchange and energy exchange with surrounding gas $[10,11]$.

The jet flow of fans in the tunnel had been regarded as turbulent free jet in many articles. The basic equations of turbulent free jet are the continuity equation and the momentum equation. They can be represented as follows:

$$
\begin{gathered}
\frac{\partial u_{i}}{\partial x_{i}}=\frac{\partial \bar{u}_{i}}{\partial x_{i}}+\frac{\partial u_{i}^{\prime}}{\partial x_{i}}=0 \\
\frac{\partial \bar{u}_{i}}{\partial t}+\bar{u}_{j} \frac{\partial \bar{u}_{i}}{\partial x_{j}}=f_{i}-\frac{1}{\rho} \frac{\partial \bar{p}}{\partial x_{i}}+\frac{1}{\rho} \frac{\partial}{\partial x_{j}}\left(\mu \frac{\partial \bar{u}_{i}}{\partial x_{j}}-\rho \overline{u_{i}^{\prime} u_{j}^{\prime}}\right)
\end{gathered}
$$

where $u_{i}$ is turbulent velocity, $\bar{u}_{i}$ is time-averaged velocity, $u_{i}^{\prime}$ is velocity fluctuation, and $f_{i}$ is mass force $(i, j=1,2,3)$.

Turbulent free jet is a common form of jet flow, and the important features are shown as follows [12].

(1) Velocity distributions in each section of the turbulent free jet are similar, the maximum velocity is axis velocity, and with the distance from the axis is farther, the velocity becomes lower.

(2) The thickness of mixing layer of turbulent free jet has linear increase with the distance.

(3) Momentum flux conservation is satisfied for the turbulent free jets and the surrounding fluid.

(4) In the zone of established flow, the boundary layer is fully developed, so the velocity obeys the Gauss distribution.

As momentum flux conservation is satisfied for each section of the turbulent free jets, when it is round jet, the momentum flux can be represented as follows:

$$
J=\int_{0}^{\infty} \rho u^{2} \cdot 2 \pi r d r=\rho u_{0}^{2} \pi r_{0}^{2}
$$

where $u_{0}$ is the velocity of nozzle exit section and $r_{0}$ is the radius of the nozzle.

The axis velocity of any section and the flow rate of any section can be, respectively, represented as follows:

$$
\begin{aligned}
& u_{m}=6.2 \frac{D}{x} \cdot u_{0}, \\
& Q=0.32 \frac{x}{D} \cdot Q_{0},
\end{aligned}
$$

where $u_{m}$ is the axis velocity, $D$ is the diameter of the nozzle, $x$ is the distance from the nozzle, and $Q_{0}$ is the flow rate.

\section{The Calculation Model}

In this paper, 3D models were established on the basis of the tunnel which is located in the central plains of China. The one-way tunnel consists of two separated channels, and each one is three-lane traffic. One of the channels is $1891 \mathrm{~m}$ long and the other is $1908 \mathrm{~m}$. The width of a channel is $14.26 \mathrm{~m}$ and the height is $7.6 \mathrm{~m}$. The cross-section of tunnel is about $88 \mathrm{~m}^{2}$. Considering that the simulation calculation will be relatively complicated if the actual situation was taken into account, the geometric model of the tunnel is simplified by ignoring the drainage channel, cable trench, and other secondary factors. The simple dimensions of the tunnel crosssection are shown in Figure 1. In the ventilation system, there are 18 individual reversible jet fans with the diameter of $900 \mathrm{~mm}$ suspended on the ceiling of the tunnel (not installed in niches). These jet fans are divided into six groups and in each group there are three jet fans. The arrangement of jet fans is shown in Figure $2(1891 \mathrm{~m})$. The width, height, and length of the channel are along $x$-coordinate, $y$-coordinate, and $z$ coordinate, respectively. 
TABLE 1: Combination modes of operational jet fans.

\begin{tabular}{ll}
\hline Operating condition & Combination modes \\
\hline 1 & Turn on 4 jet fans of group 1 and group 3 (turn off the middle one of each group) \\
2 & Turn on the middle jet fan of each group, from group 1 to group 4 \\
3 & Turn on 4 jet fans of group 1, group 2, and group 3 (turn on jet fans on left and right \\
& sides of group 1, and turn on the middle jet fans of other two groups) \\
4 & Turn on 4 jet fans of group 1, group 3, and group 4 (turn on jet fans on left and right \\
& sides of group 1, and turn on the middle jet fans of other two groups)
\end{tabular}

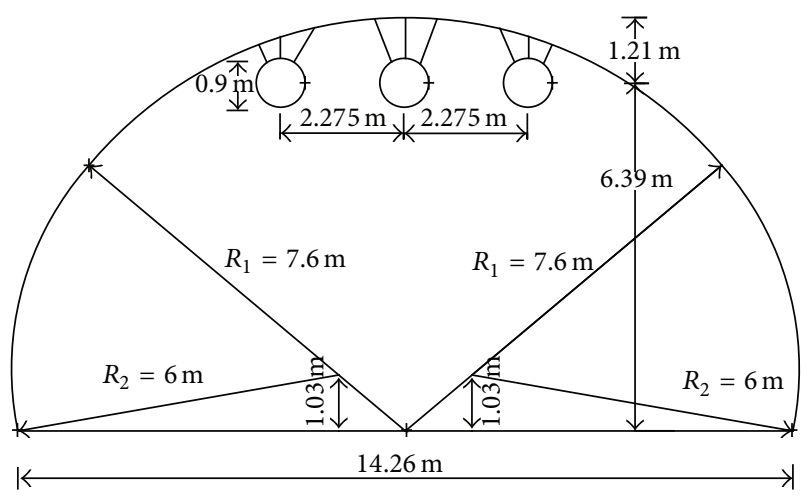

FIGURE 1: Simple dimensions of the tunnel cross-section.

\begin{tabular}{|c|c|c|c|c|c|c|}
\hline 1 & 2 & 3 & & 4 & 5 & 6 \\
\hline$\square$ & $\square$ & $\square$ & \multirow{3}{*}{$\Rightarrow$} & $\square$ & $\square$ & $\square$ \\
\hline$\square$ & $\square$ & 口 & & $\square$ & $\square$ & $\square$ \\
\hline$\square$ & 口 & ص & & $\square$ & $\square$ & 口 \\
\hline $200 * 1$ & $0 \quad 15$ & & 910 & & 0,15 & 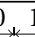 \\
\hline
\end{tabular}

Figure 2: Layout of the jet fans in the tunnel used as case study showing the relative positions of the jet fans and portals $(\mathrm{m})$.

Generally, the number of operational fans is calculated by designers according to the norm. The design instructions point out that only four operational jet fans will ensure the air quality inside the tunnel when the speed of the vehicles is between $40 \mathrm{~km} / \mathrm{s}$ and $60 \mathrm{~km} / \mathrm{s}$. In the study present here, the numerical simulations were carried out according to the normal traffic condition with 4 jet fans operating. As the series mode is often designed on the basis of designers' experience, in this paper, four different combination modes have been designed. The four combination modes are shown in Table 1.

The tunnel length is so long that the computational cost will be large if the models were built according to the prototype of the tunnel. In order to reduce the computational cost, the symmetry of the solution across the longitudinal plane was considered. So, the models were built based on the right half part of the tunnel (the cross-section of tunnel is symmetrical). As the cross-section of the tunnel is irregular, unstructured meshes have been used in the computational domain. The calculated domain has been divided into a plurality of subregions. In the region near the jet fans, the meshes are intensive, while, in the region far away from the jet fans, the meshes are coarse. The meshes of different tunnel cross-sections are shown in Figure 3. The total number of meshes throughout the computational domain is more than five hundred thousand. The calculations in the four models were cold-flow steady state. The standard $k-\varepsilon$ turbulent model was applied to the $3 \mathrm{D}$ models and the second order upwind discretization was used for other terms except for pressure term which was dealt with the standard discretization. The SIMPLEC algorithm was used for the coupling between velocity and pressure. In addition, the boundary conditions were specified as follows. The nonslip stationary wall boundary condition was applied on the walls of tunnel and jet fans. The pressure inlet boundary and pressure outlet boundary were prescribed at tunnel inlet and tunnel outlet, respectively, and the relative pressure was set as $0 \mathrm{~Pa}$. The fan inlet and fan outlet were specified as velocity inlet boundary and velocity outlet boundary, respectively, and the velocity was set as $30.7 \mathrm{~m} / \mathrm{s}$ which is the issue velocity of jet fan with the diameter of $900 \mathrm{~mm}$.

\section{The Numerical Simulation Results and Analysis}

4.1. The Analysis of the Jet Mode in the Tunnel. The jet flow inside tunnel is quite complex due to the restriction of various factors. In order to validate the jet mode inside tunnel in this paper, the space within $200 \mathrm{~m}$ away from the fan outlet is selected as the research region. The variation of axis velocity along the tunnel length direction is shown in Figure $4(x=$ $0 ; y=6.39$; velocity profile along $z$-coordinate direction). The results of numerical simulation are compared with the theoretical value which is calculated based on formula (3).

The blue line in Figure 4 is the theoretical curve and the red line is the curve obtained from the simulation results. Comparing the two curves, it can be seen that the velocity decay trend of numerical simulation curve is consistent with that of theoretical curve, although there is certain difference in magnitude. Within $50 \mathrm{~m}$ away from the fan outlet, the velocity drops sharply with the increasing of distance; then, in the following $50 \mathrm{~m}$, the velocity drops slowly as the distance increases. When the distance from the fan outlet is more than $100 \mathrm{~m}$, the difference of velocity is slight; in other words, it essentially remains the same.

The theoretical length of the zone of flow establishment is expressed as

$$
L_{0}=6.2 \cdot D
$$




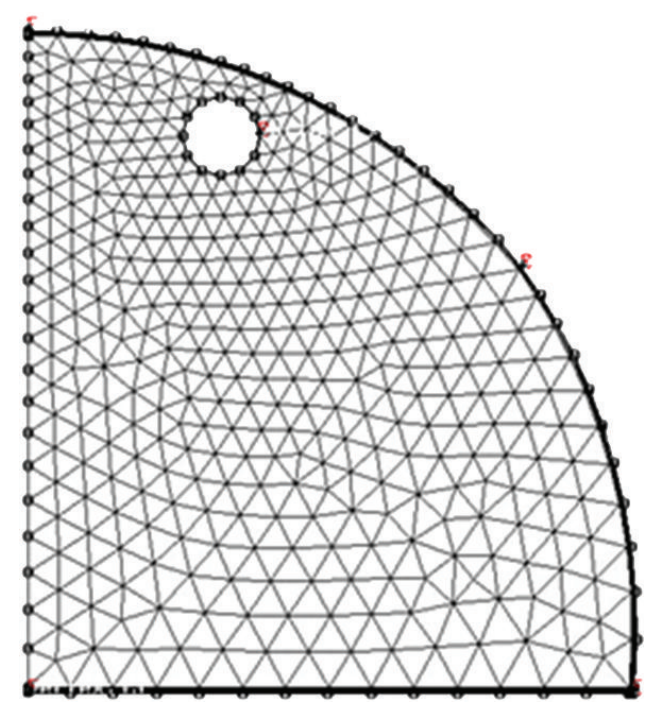

(a)

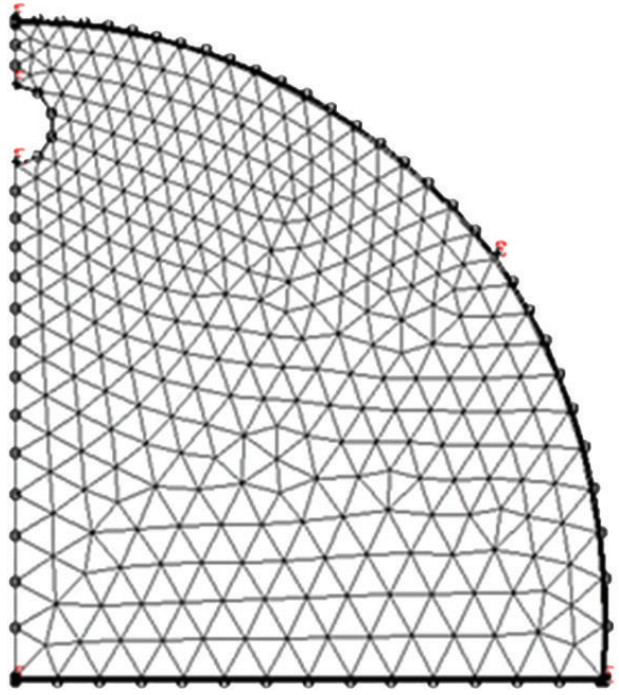

(b)

Figure 3: Different meshes used for half of the tunnel cross-sections (the left cross-section shows the domain containing jet fan pairs, and the right cross-section shows the domain containing single jet fan).

where $L_{0}$ is the length of the zone of flow establishment and $D$ is the diameter of jet nozzle. So, there is a horizontal line about $6 \mathrm{~m}$ on the theoretical curve which represents the length of zone of flow establishment. For the reason that, in the enclosed tunnel, the limit of space and the wall friction would lead to the insufficient development of turbulent free jet. Hence, on the numerical simulation curve, there is no horizontal line and the velocity drops from the exit of jet fan. Within $15 \mathrm{~m}$ away from the fan outlet, the theoretical velocity is higher than that of numerical simulation, while when the distance from the fan outlet is more than $15 \mathrm{~m}$, it is reversed and the velocity of numerical simulation overshoot the theoretical velocity. The reasons for the generation of this phenomenon can be described as follows: after the airflow spraying out of jet fan, the entrainment will occur between the jet flow and surrounding gas. As the jet flow spreads forward, more and more free-stream air is entrained into the high-speed jet flow. With the entrainment downstream, the velocity gradually decreases and finally is evenly distributed at the cross-section. The exchange of momentum between jet flow and surrounding gas leads to the rise of pressure, and then the movement of air flow is driven by pressure gradient. Accordingly, from Figure 4, we can conclude that the jet flow inside tunnel has a little difference with the turbulent free jet, but it can still be regarded as the turbulent free jet.

4.2. The Analysis of Twin-Jet. In the 3D simulation models, there exists twin-jet in the tunnel. According to the flow characteristics, the twin-jet region can be divided into converging region and combined region. In converging region, the entrainment between twin-jet and free-stream air leads to the spread of outer edge. Then, the outer edges of the two jets begin to interact with each other at the centerline. Eventually, the two jets merge into a single one in combined region.

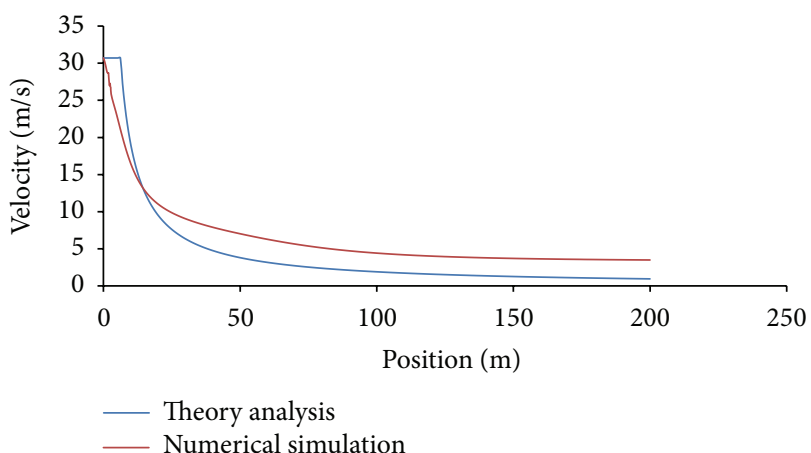

FIGURE 4: The comparison of the theoretical velocity and calculated velocity of jet changing with the distance from jet fan (at the height of the axis of jet fan).

The change process of twin-jet from converging region to combined region is illustrated in Figure 5.

Figure 5 shows the horizontal velocity profiles at different axial location for the condition of $z=6.39 \mathrm{~m}$. The different axial location, in other words, is the different axis distance from the fan outlet and $z$ represents the height from ground. At the early stage, the velocity profile exhibits the typical characteristic of twin-jet. As the jets spread downstream, the core velocity gradually decreases. Within $10 \mathrm{~m}$ away from fan outlet, the peak velocity drops sharply, from $30.7 \mathrm{~m} / \mathrm{s}$ to $17 \mathrm{~m} / \mathrm{s}$. The velocity of the region around centerline has no change, still below $4 \mathrm{~m} / \mathrm{s}$, whereas it can be seen that the outer edge of each jet has expanded. When the distance from fan outlet is more than $10 \mathrm{~m}$, the outer edges of two jets gradually merge with each other. At the same time, the velocity of centerline gradually increases. In the following $40 \mathrm{~m}$, the peak velocity gradually drops and eventually becomes unobvious. 


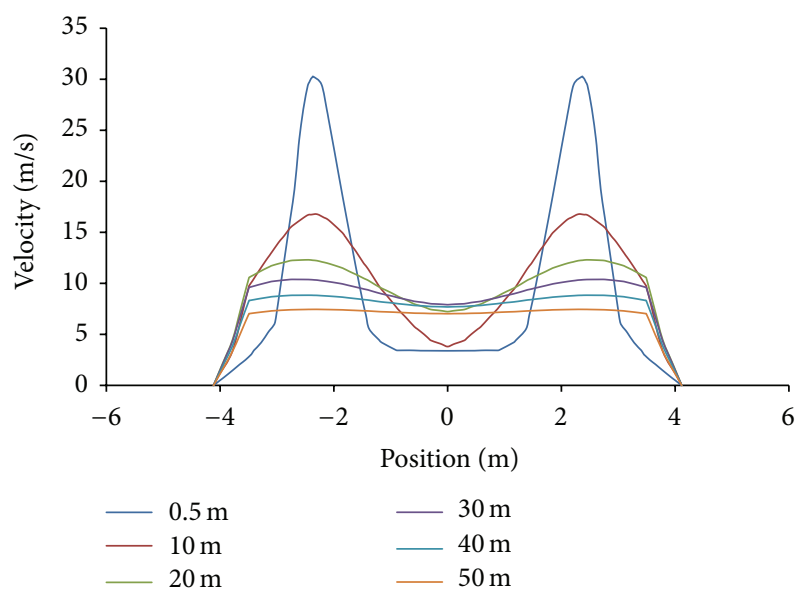

FIGURE 5: Horizontal velocity profiles for various distances from jet fan pair at the height of fan axis.

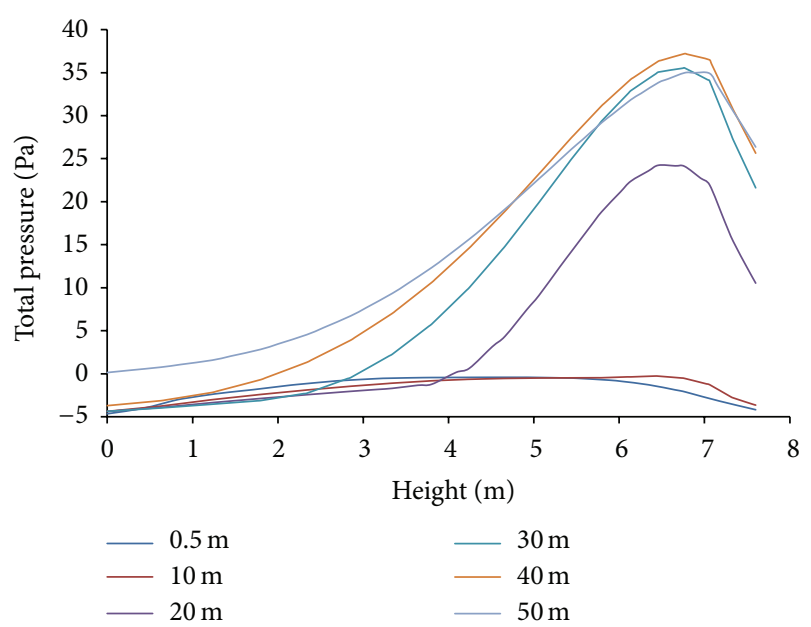

FIGURE 6: Pressure profiles along the vertical direction for various distances from the jet fan pair (in the central axis of the tunnel crosssection).

At the position of $20 \mathrm{~m}$ away from fan outlet, the peak velocity is $12 \mathrm{~m} / \mathrm{s}$, while, at the position of $50 \mathrm{~m}$ away from fan outlet, there is no obvious peak velocity because the velocity is substantially evenly distributed. In the region more than $20 \mathrm{~m}$ away from fan outlet, the velocity of the region around centerline remains $7 \sim 8 \mathrm{~m} / \mathrm{s}$.

The pressure variation is very important for understanding the characteristic of the jet flow. Figure 6 shows the pressure profiles in the central axis of different cross-sections with various distances from fan outlet. Here, the abscissa stands for the height from ground. From this figure, it can be seen that there exists negative pressure region. Within $10 \mathrm{~m}$ away from fan outlet, from ground to the roof of tunnel, the air pressure is lower than $0 \mathrm{~Pa}$ because of the entrainment and mutual interference of twin-jet. As the jet boundary gradually expands to the lower space of tunnel, the negative pressure region becomes more and more small. At the position of $20 \mathrm{~m}$, $30 \mathrm{~m}$ and $40 \mathrm{~m}$ away from fan outlet, the upper bounds of negative pressure region are $4 \mathrm{~m}, 3 \mathrm{~m}$, and $2 \mathrm{~m}$, respectively. When the distance from fan outlet is more than $50 \mathrm{~m}$, there is no negative pressure region.

In order to verify the reasonableness of numerical calculation, it is necessary to compare the calculated data with the experimental data. In the literature [9], Colella et al. had measured the longitudinal air velocities of various points in the condition with only 5th jet fan pair operating in the West tunnel of the Dartford tunnels in UK. These points are distributed in six different cross-sections, at $20 \mathrm{~m}$ intervals, starting $20 \mathrm{~m}$ downstream from the jet fan discharge surface. As the tunnel size and fan model in literature [9] are different from that we used in numerical simulation, we just compared the air velocity change trend along the longitudinal direction of two cases. From the various measured points in the literature [9], we picked out two points in central line of every cross-section with the heights of $2.75 \mathrm{~m}$ and $4.5 \mathrm{~m}$ from ground (the location of the two points also can be regard as about $2.35 \mathrm{~m}$ and $0.6 \mathrm{~m}$ below the height of fan axis). And, from the calculated data, we picked out the points at the same location (in the central line of cross-section and about $2.35 \mathrm{~m}$ and $0.6 \mathrm{~m}$ below the height of fan axis) in different cross-sections with $20 \mathrm{~m}, 40 \mathrm{~m}, 60 \mathrm{~m}, 80 \mathrm{~m}, 100 \mathrm{~m}$, and $120 \mathrm{~m}$ downstream from the jet fan discharge surface, respectively. The comparison of change trend of velocities between the measured data and calculated data are shown in Figure 7(a) and Figure $7(\mathrm{~b})$.

From Figure 7(a) and Figure 7(b), we can conclude that the velocity change trend of numerical simulation is consistent with that of experimental measurements. It can be seen from Figure 7(a) that, at the location of $0.6 \mathrm{~m}$ below the height of fan axis, both the experimental velocity and calculated velocity decrease as the distance from the jet fan discharge surface increases, while, from Figure 7(b), it can be concluded that as the distance from the jet fan discharge surface increases, for both the experimental velocity and calculated velocity, there exists velocity fluctuation in a small range. Therefore, the results of numerical calculation will be able to reflect the real change trend of air velocity in the tunnel.

4.3. The Comparison between Twin-Jet and Single Jet. The movement of air inside tunnel under the conditions of twinjet is different from that of single jet. Accordingly, the velocity distribution and pressure distribution in the two conditions will be different.

Figure 8 shows the velocity distributions in the central axis of tunnel cross-section of the two cases with the distance from the fan outlet are $50 \mathrm{~m}, 100 \mathrm{~m}$, and $150 \mathrm{~m}$. It can be concluded that, in both cases, the velocity difference between the upper space (above $3 \mathrm{~m}$ ) and lower space (below $3 \mathrm{~m}$ ) is smaller as distance increases. At the position of $50 \mathrm{~m}$ away from fan outlet, the velocity difference between the upper space and lower space of tunnel is $5 \sim 6 \mathrm{~m} / \mathrm{s}$. In the following $100 \mathrm{~m}$, the velocity difference drops to lower than $1 \mathrm{~m} / \mathrm{s}$. When the distance from fan outlet is more than $100 \mathrm{~m}$, the velocity variation is slight and the velocities are evenly distributed along tunnel height direction. At the position of $50 \mathrm{~m}$ away from fan outlet, in upper space, the velocity in the 


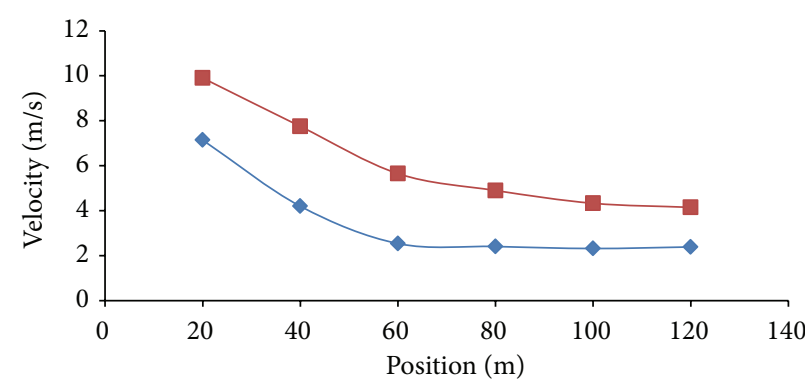

(a)

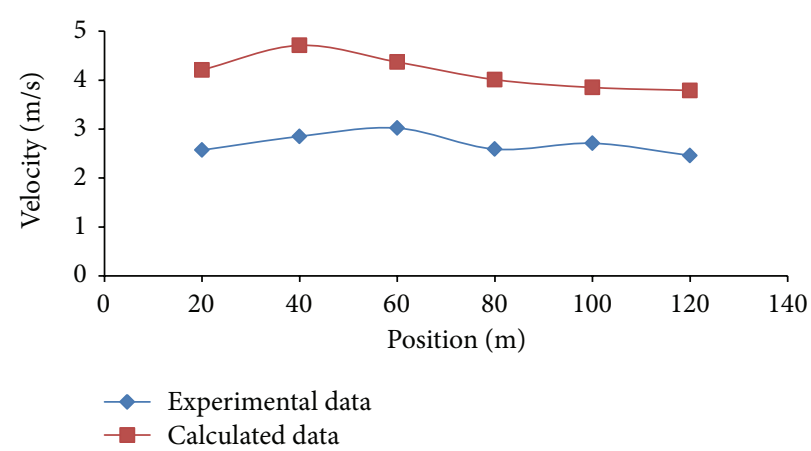

(b)

FIGURE 7: The comparison of the change trend of velocities between numerical simulation and experimental measurements (all the points in (a) are at the location of $0.6 \mathrm{~m}$ below the height of fan axis; all the points in (b) are at the location of $2.35 \mathrm{~m}$ below the height of fan axis).

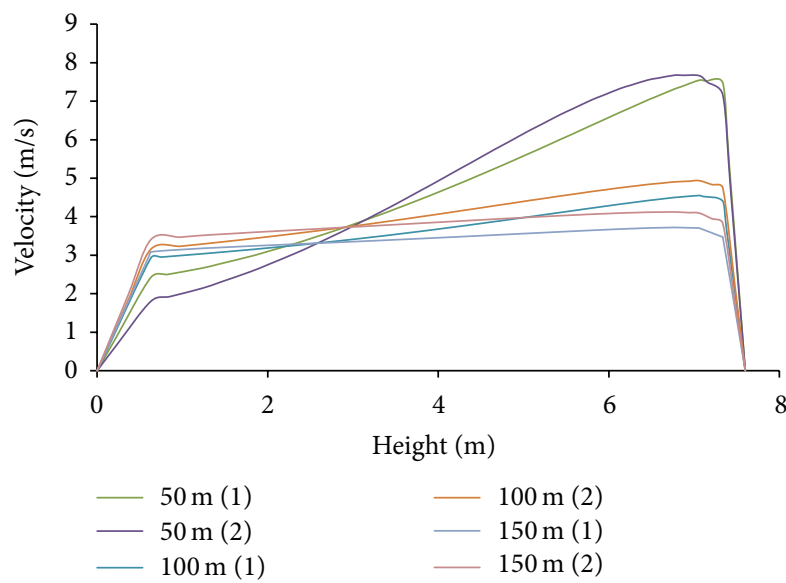

FIGURE 8: The comparison of vertical velocity profiles for various distances from single jet fan and jet fan pair (in the central axis of the tunnel cross-section).

condition with two operational fans is higher than that with one operational fan, while, in lower space, it is reversed. At the positions of $100 \mathrm{~m}$ and $150 \mathrm{~m}$ away from fan outlet, whether in the upper space or in the lower space, the velocity in the condition with two operational fans is a little higher than that with one operational fan. Figure 9 shows the velocity distributions of the two cases at the fan axis height with the distance from the fan outlet are $50 \mathrm{~m}, 100 \mathrm{~m}$, and $150 \mathrm{~m}$. It is known that, at the position of $100 \mathrm{~m}$ away from fan outlet, the twin-jet has completely merged into one and the peak velocity has moved to the centerline. No matter how far it is from the fan outlet $(50 \mathrm{~m}, 100 \mathrm{~m}$ or $150 \mathrm{~m})$, the velocity in the condition with two operational fans is higher than that with one operational fan. It can be concluded from Figure 8 and Figure 9 that, compared to single jet, twin-jet is more conducive to increasing the wind speed inside tunnel.

From Figure 10, we can know that, no matter how far it is from the fan outlet, the pressure of single jet is a little higher than that of twin-jet, while, from Figure 11, it can be seen that, at the position of $50 \mathrm{~m}$ away from fan outlet, the pressure of single jet is higher than that of twin-jet only in the

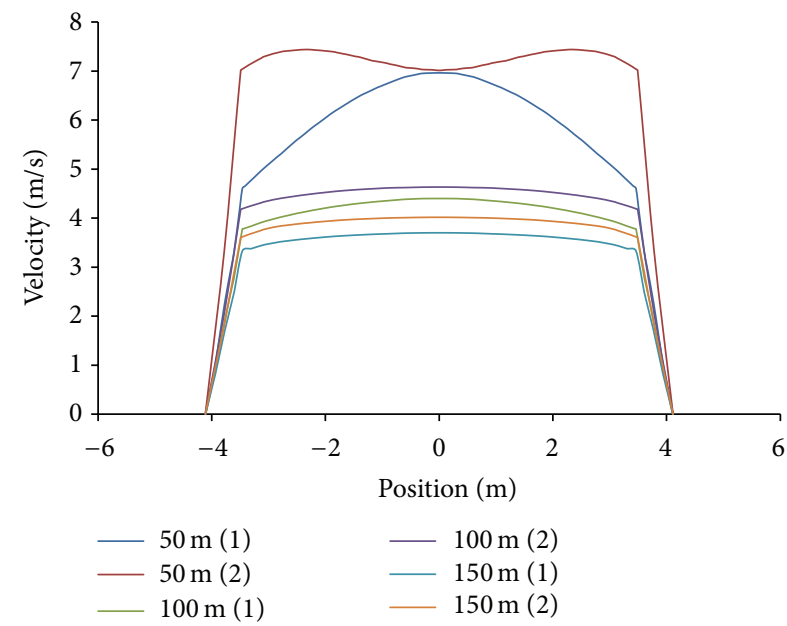

FIGURE 9: The comparison of horizontal velocity profiles for various distances from single jet fan and jet fan pair (at the height of fan axis).

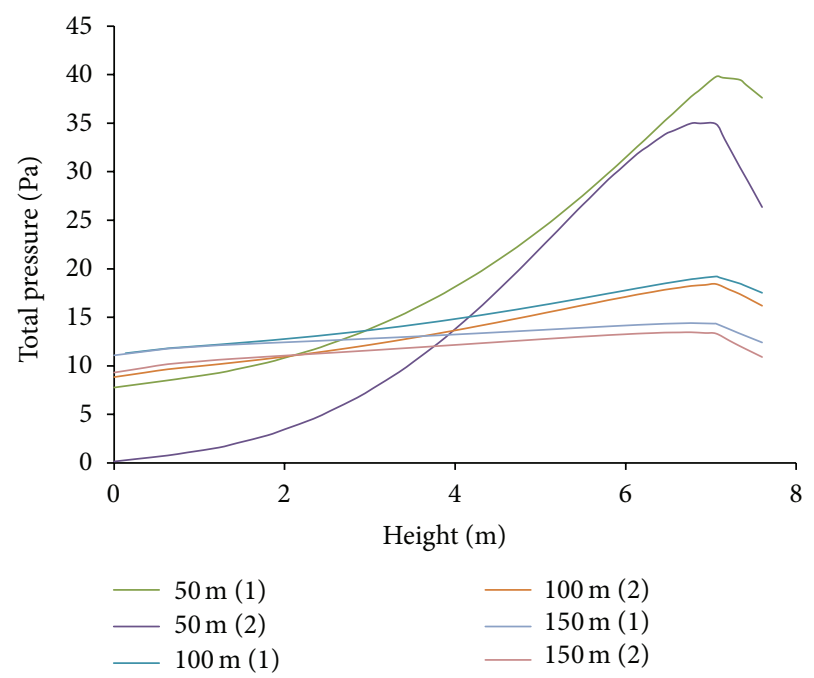

FIGURE 10: The comparison of vertical pressure profiles for various distances from single jet fan and jet fan pair (in the central axis of the tunnel cross-section). 


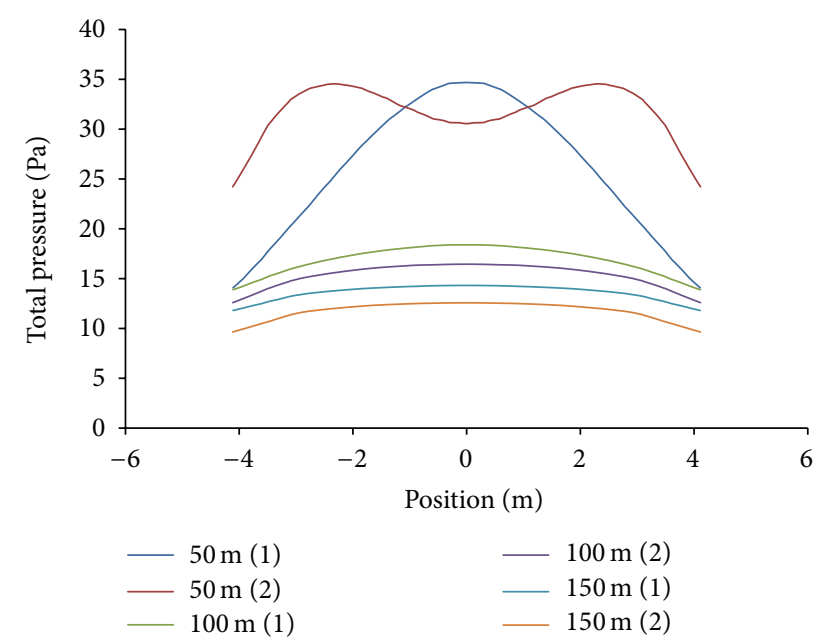

FIGURE 11: The comparison of horizontal pressure profiles for various distances from single jet fan and jet fan pair (at the height of fan axis).

center space; however, apart from center space, the pressure of twin-jet is higher than that of single jet. That is because, at jet axis, the motion of fluids is more intense. From Figure 10 and Figure 11, it can be seen that in both cases the pressure gradually decreases as the distance from fan outlet increases.

4.4. The Analysis of Different Operating Conditions. The pressure inside tunnel has increased when the jet is fully developed. In the region that the distance from the fan outlet is less than $100 \mathrm{~m}$, the pressure has reached the top and the movement of air is driven by pressure gradient. In general, the larger the pressure gradient is, the greater the wind speed is. And, in a certain range, it is more conductive in discharging waste gas when the wind speed is relatively high. Since the tunnel ventilation system is composed of series of fans, the distance between adjacent two groups and the number of operational fans will affect the air pressure and wind speed inside tunnel. Therefore, we analyzed the velocity distribution and pressure distribution of four combination modes and chose the best combination mode. Thus, the section $1.5 \mathrm{~m}$ high from ground is selected for research. The comparison between first operating condition and second operating condition is shown in Figure 12.

Figure 12(a) shows the velocity distribution along the tunnel on the center line of the plane $1.5 \mathrm{~m}$ high from ground. Figure 12(b) shows the pressure distribution at the same position. It can be seen that the gas pressure and wind speed of second operating condition are lower than those of first operating condition. Although there are only two groups of fans in series in first operating condition, the effect of pressure-rise and velocity-rise is better than that of second operating condition. In Figure 12(a), within $100 \mathrm{~m}$ away from fan outlet of each group, the wind speed suddenly drops to below $2.5 \mathrm{~m} / \mathrm{s}$ and then recovers to above $3 \mathrm{~m} / \mathrm{s}$. The difference between maximum velocity and minimum velocity is $1.8 \mathrm{~m} / \mathrm{s}$ in first operational condition, while the velocity difference is $1.2 \mathrm{~m} / \mathrm{s}$ in second operational condition. The velocity difference in the mode of twin-jet is larger than that of single jet. Except for the region mentioned above, the wind speed is essentially unchanged in the other region of the tunnel. Near the entrance of tunnel, the velocity of first operating condition is $0.3 \mathrm{~m} / \mathrm{s}$ higher than that of second operating condition, and so it is in the middle of tunnel, while near the exit of tunnel, the velocity difference of two operating conditions is $0.4 \mathrm{~m} / \mathrm{s}$. In Figure $12(\mathrm{~b})$, it can be known from the curve that the pressure-rise in the mode with double paralleled fans is higher than that with two jet fans in series. In addition, in the second condition, the distance between last two groups of fans is so far that the effect of pressure-rise is not continuous. As a consequence, the effect of pressure-rise in the mode with two groups of double paralleled fans in series is better than that with four groups of single fan in series. In the middle of tunnel, the pressure of first operating condition is $10 \mathrm{~Pa}$ higher than that of second operating condition, whereas near the exit of tunnel, the pressure difference of two conditions is only $2 \mathrm{~Pa}$. In conclusion, it can be confirmed that the ventilation performance depends on both the series mode and the number of fans in series.

Figure 13(a) shows the velocity distribution along the tunnel on the center line of the plane $1.5 \mathrm{~m}$ high from the ground. Figure 13(b) shows the pressure distribution at the same position. From Figure 13(a), we can conclude that, in the novel series modes, the velocity and pressure inside tunnel will change with the separation of adjacent groups of fans. In third operating condition, the distance between adjacent groups of fans is short, the velocity difference of maximum velocity and minimum velocity in first "velocity oscillation" is $1.9 \mathrm{~m} / \mathrm{s}$, in second and third "velocity oscillation" the velocity difference is $0.8 \mathrm{~m} / \mathrm{s}$. In fourth operating condition, the distance between adjacent groups of fans is long and the velocity difference in first, second, and third "velocity oscillation" is $1.8 \mathrm{~m} / \mathrm{s}, 1.1 \mathrm{~m} / \mathrm{s}$, and $1.1 \mathrm{~m} / \mathrm{s}$, respectively. On the other hand, near the entrance of tunnel, the velocity of third operating condition is only $0.1 \mathrm{~m} / \mathrm{s}$ higher than that of fourth operating condition, and so it is in the middle of tunnel. Near the exit of tunnel, the velocity of third operating condition is $0.2 \mathrm{~m} / \mathrm{s}$ higher than that of fourth operating condition. It can be seen from Figure 13(b) that the pressure inside tunnel of third operating condition is higher than that of fourth operating condition. In the middle of tunnel, the pressure of third operating condition is $8 \mathrm{~Pa}$ higher than that of fourth operating condition, and near the exit of tunnel, the pressure difference of two operating conditions is $1 \mathrm{~Pa}$. That is because, in third operating condition, the pressure inside tunnel has been continuously enhanced for three times in a short distance, while, at the same distance, the pressure only has been continuously enhanced twice in fourth operating condition. Although the pressure is enhanced at the position of $1500 \mathrm{~m}$ in fourth operating condition, the pressure of fourth operating condition is still lower than that of third operating condition. Consequently, it can be concluded that it is more conductive in enhancing the velocity and pressure inside tunnel when the distance between adjacent groups of fans is short.

Reviewing Figure 13(a) and Figure 13(b), it is not difficult to find that it is more conductive in enhancing the velocity 


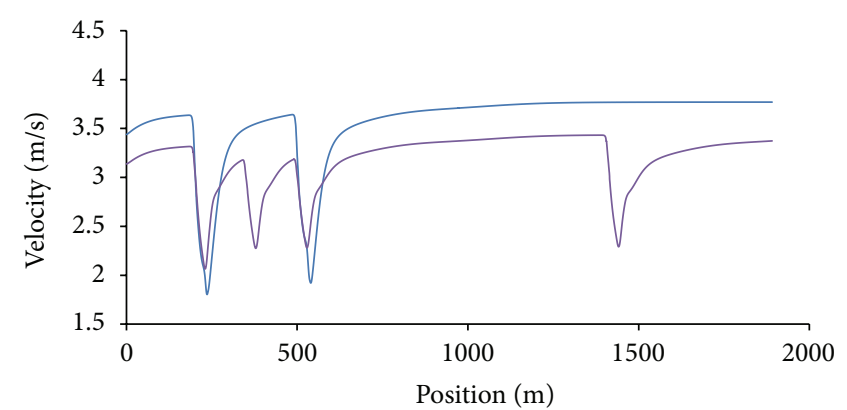

(a)

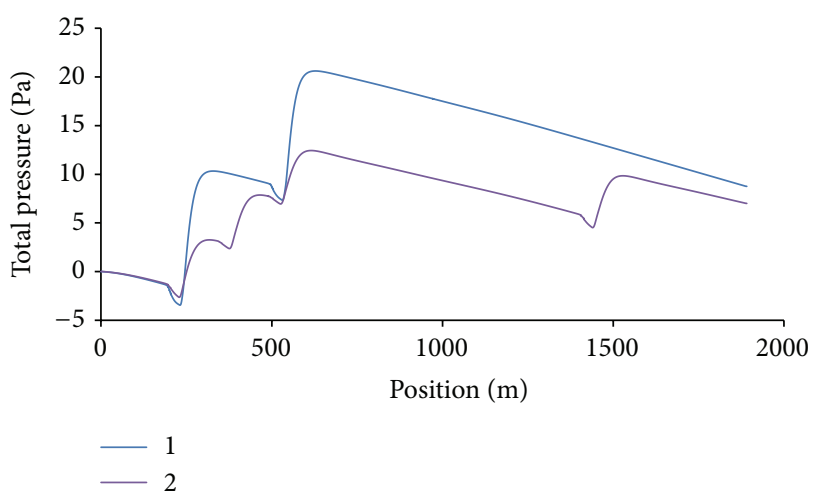

(b)

FIGURE 12: The comparison of velocity profiles and pressure profiles along the longitudinal direction of first operating condition and second operating condition (the velocity profiles and pressure profiles obtained from the section $1.5 \mathrm{~m}$ high from the ground).

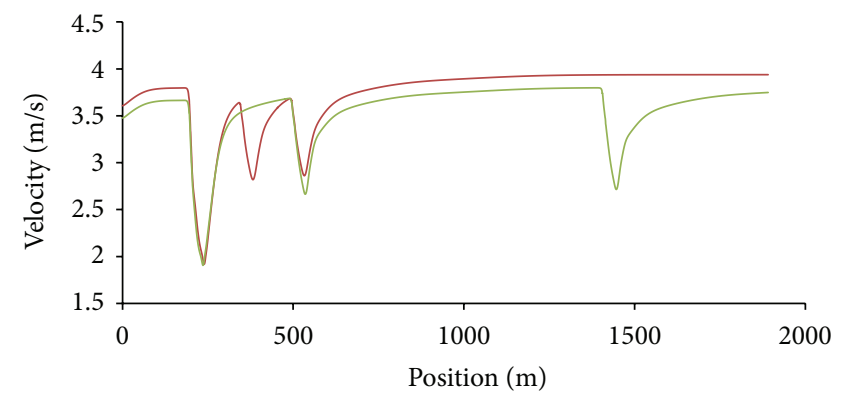

(a)

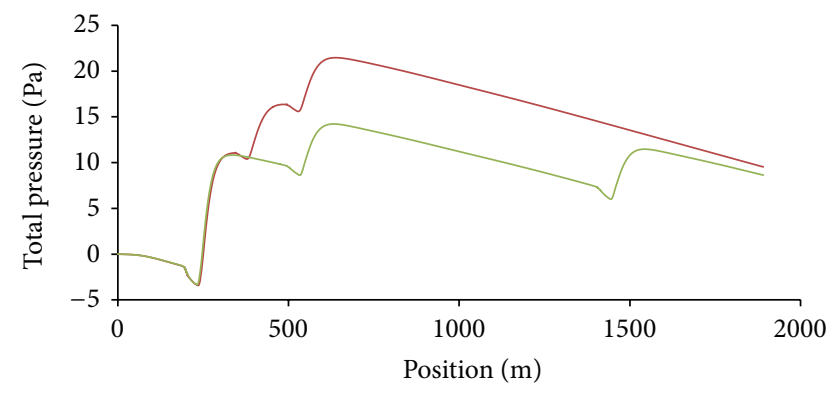

$-3$

(b)

FIGURE 13: The comparison of velocity profiles and pressure profiles along the longitudinal direction of third operating condition and fourth operating condition (the velocity profiles and pressure profiles obtained from the section $1.5 \mathrm{~m}$ high from the ground).

and pressure inside tunnel when most operational fans are located near the tunnel entrance. Since the operational fans of first operating condition and third operating condition are all located near the tunnel entrance, we should make a comparison between the traditional series mode and novel series mode to choose the better one.

The Figure 14(a) and Figure 14(b) present that, in first operating condition, either the pressure or the velocity is lower than that in third operating condition. In Figure 14(a), near the entrance of tunnel, the velocity of third operating condition is $0.2 \mathrm{~m} / \mathrm{s}$ higher than that of first operating condition, and when the distance from the entrance of tunnel is more than $500 \mathrm{~m}$, the velocity of third operating condition is $0.15 \mathrm{~m} / \mathrm{s}$ higher than that of first operating condition. So, compared to the traditional series mode, the velocity can be increased about $5.7 \%$ with the novel series mode. From Figure 14(b), it can be seen that the pressure of third operating condition is continuously enhanced for three times within $500 \mathrm{~m}$, while in first operating condition it is continuously enhanced twice at the same distance. When the distance from the entrance of tunnel is more than $500 \mathrm{~m}$, the pressure of third operating condition is a little higher than that of first operating condition. In conclusion, the third operating condition is more beneficial in exhausting waste gas in the light of calculation data.

\section{Conclusion}

Numerical simulations were conducted to research the characteristics of air flow in a tunnel. The jet flow inside tunnel is a little different from the turbulent free jet because of the wall friction and the constraint of space. But the change trend of axial velocity obtained from simulation results agrees quite well with turbulent free jet theory. In this paper, four calculation models were carried out to investigate the velocity distribution and pressure distribution inside tunnel with different combination modes of jet fans. From the simulation results, it can be concluded that the ventilation performance mainly depends on the series mode and the separation of adjacent groups of fans. The novel combination mode which consists of double paralleled fans and single fan is regarded as the optimum combination mode. When most operational fans are located near the tunnel entrance, it is more conductive in increasing the velocity and pressure inside tunnel. 


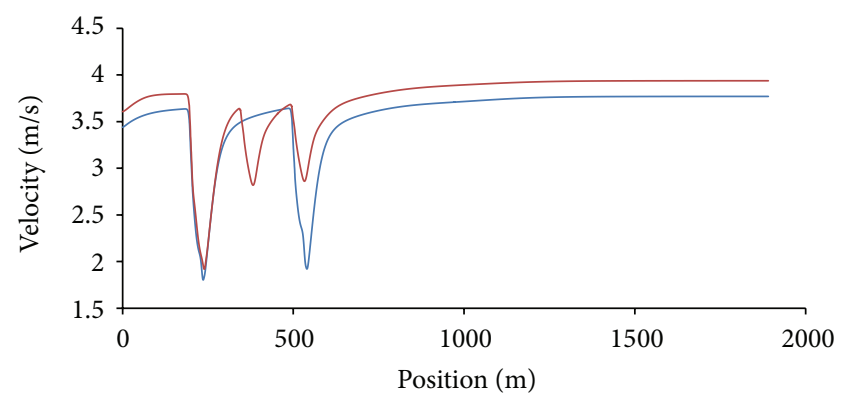

(a)

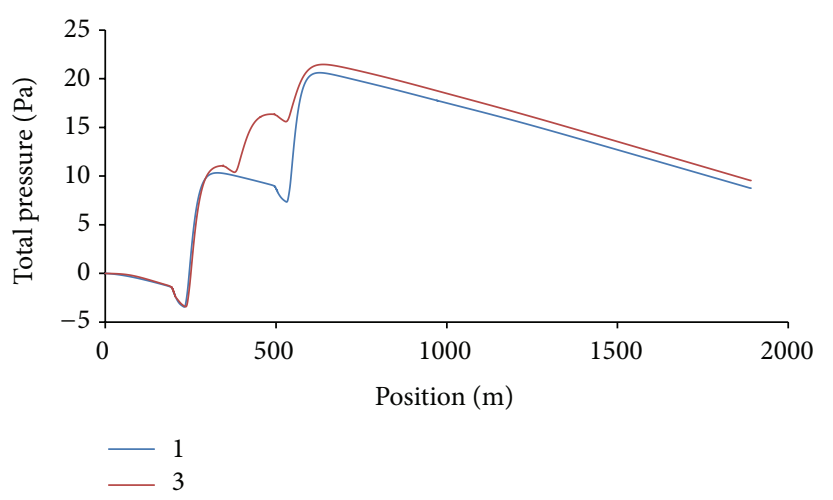

(b)

FIGURE 14: The comparison of velocity profiles and pressure profiles along the longitudinal direction of first operating condition and third operating condition (the velocity profiles and pressure profiles obtained from the section $1.5 \mathrm{~m}$ high from the ground).

In conclusion, it is needed to consider various factors when choosing the appropriate series mode in ventilation system.

\section{Conflict of Interests}

The authors declare that there is no conflict of interests regarding the publication of this paper.

\section{Acknowledgments}

This paper is financially supported by Natural Science Foundation of China (Grant no. 51174170) and National Science and Technology Major Project of China under Grant no. 2011ZX05013004. The authors are grateful to Professor Jianjun Liu for his constructive comments on this paper.

\section{References}

[1] F. Colella, G. Rein, R. Borchiellini, and J. L. Torero, "A novel multiscale methodology for simulating tunnel ventilation flows during fires," Fire Technology, vol. 47, no. 1, pp. 221-253, 2011.

[2] J. Ji, Z. H. Gao, C. G. Fan, and J. H. Sun, "Large Eddy Simulation of stack effect on natural smoke exhausting effect in urban road tunnel fires," International Journal of Heat and Mass Transfer, vol. 66, pp. 531-542, 2013.

[3] H. Ohashi, A. Mizuno, I. Nakabori, and M. Ueki, "A new ventilation method for the Kan-etsu road tunnel," in Proceedings of the 4th International Symposium on the Aerodynamics \& Ventilation of Vehicle Tunnels: Held at York, England, BHRA Fluid Engineering, vol. 4, p. 31, 1982.

[4] E. Rohne, "The friction losses on walls caused by a row of four parallel jet flows," in Proceedings of the 6th ISAVVT, BHRA Fluid Engineering, pp. 151-164, Durham, UK, 1988.

[5] M. L. Gao and J. M. Wu, "Regulating action and analysis of jet fans in the longitudinal ventilation of vehicle tunnel," Journal of Lanzhou Railway Institute, vol. 17, no. 1, pp. 88-94, 1998.

[6] H. S. Zhong, W. M. Li, and J. M. Xu, "A study on the ventilation control modes in one-way traffic tunnels," Modern Tunneling Technology, vol. 42, no. 2, pp. 76-80, 2005.
[7] Y. D. Wang and Y.X. Xia, "Numerical simulation of longitudinal ventilation of highway tunnel," China Journal of Highway and Transport, vol. 15, no. 1, pp. 82-85, 2002.

[8] F. Colella, G. Rein, R. Carvel, P. Reszka, and J. L. Torero, "Analysis of the ventilation systems in the Dartford tunnels using a multi-scale modelling approach," Tunnelling and Underground Space Technology, vol. 25, no. 4, pp. 423-432, 2010.

[9] F. Colella, G. Rein, V. Verda, and R. Borchiellini, "Multiscale modeling of transient flows from fire and ventilation in long tunnels," Computers and Fluids, vol. 51, no. 1, pp. 16-29, 2011.

[10] J. L. Zheng, "The simulation of tunnel ventilation system and the conclusion," Underground Engineering and Tunnels, no. 1, pp. 41-45, 1997.

[11] D. F. Chen, "An overview of ventilation design of long tunnels," Underground Engineering and Tunnels, no. 3, pp. 34-37, 1995.

[12] Z. Y. Dong, Jet Mechanics, Science Press, New South Wales, Australia, 2005 


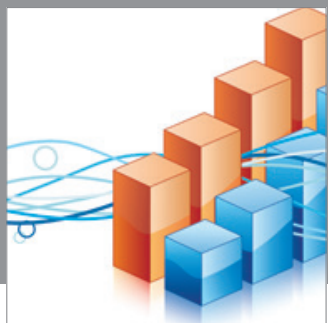

Advances in

Operations Research

mansans

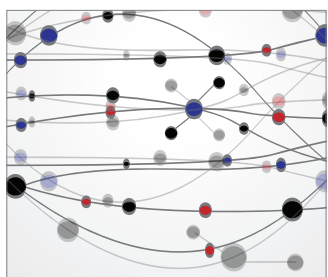

The Scientific World Journal
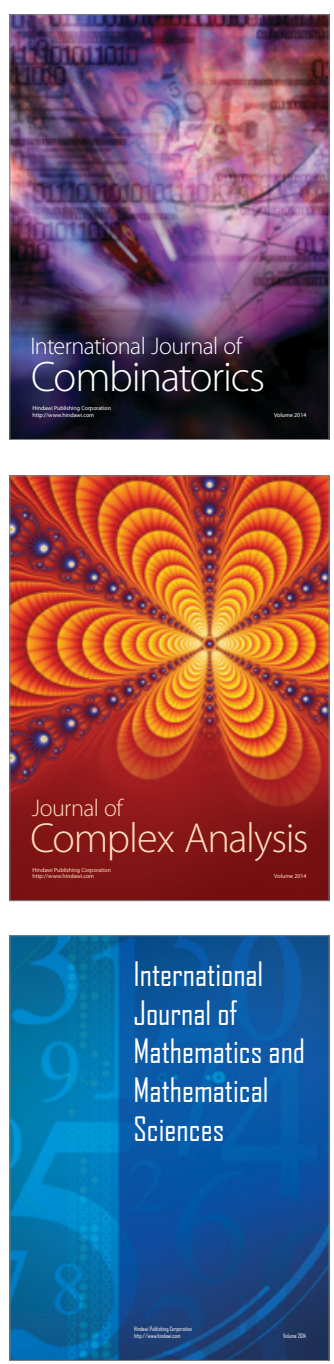
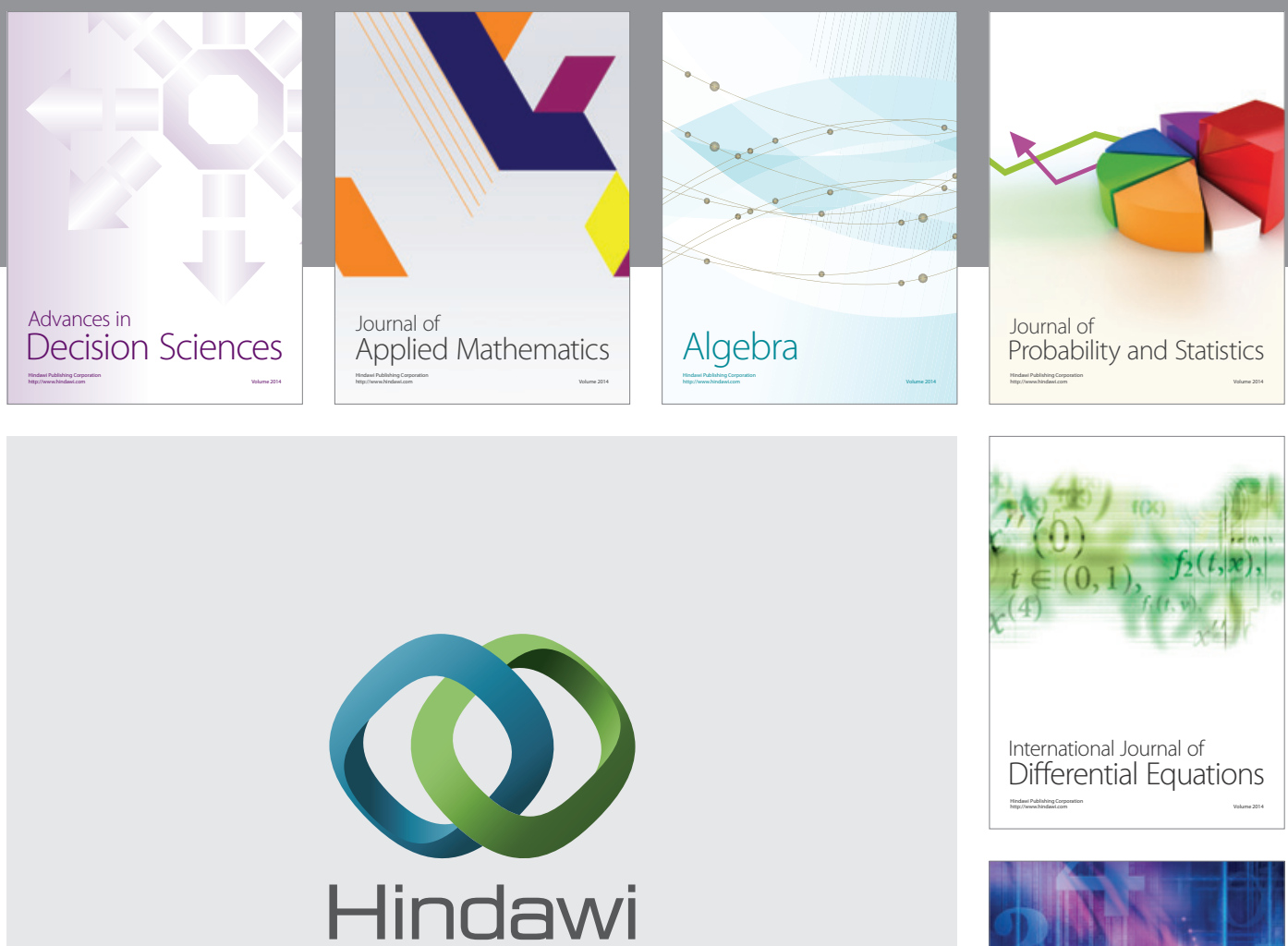

Submit your manuscripts at http://www.hindawi.com
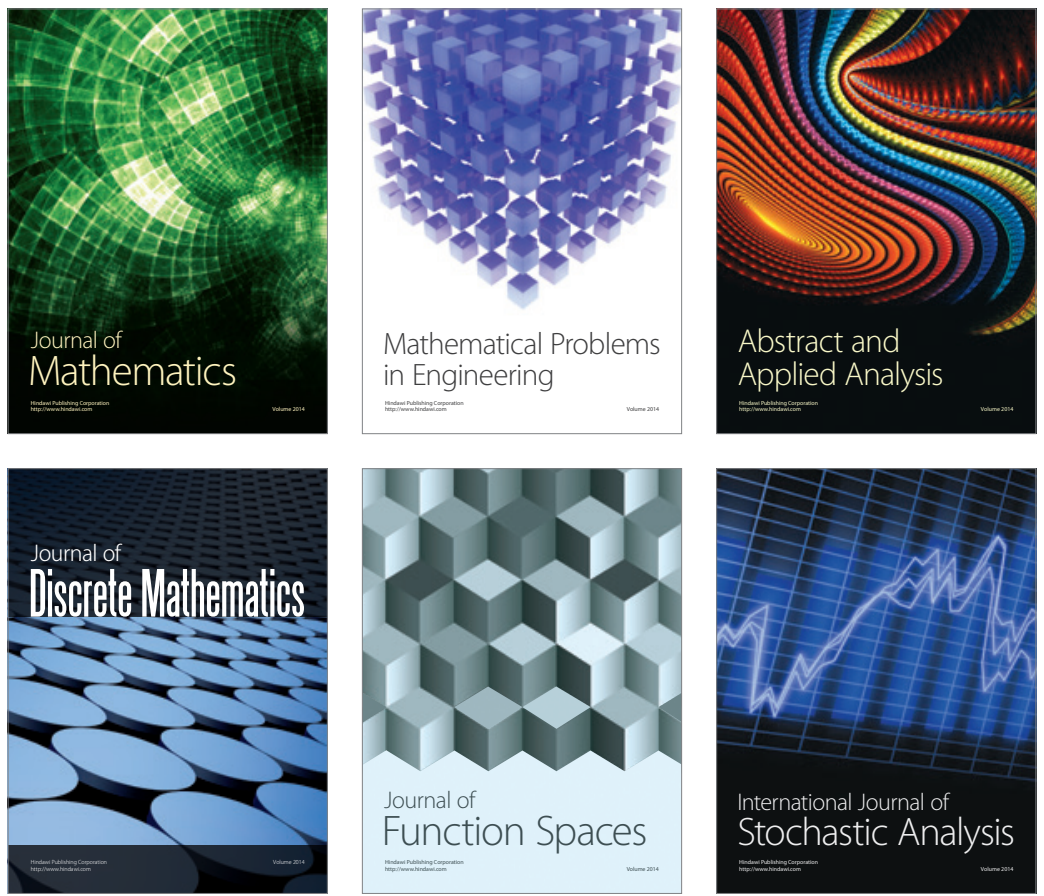

Journal of

Function Spaces

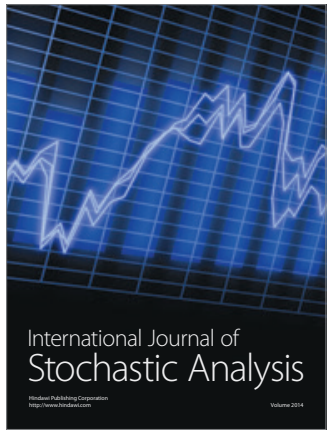

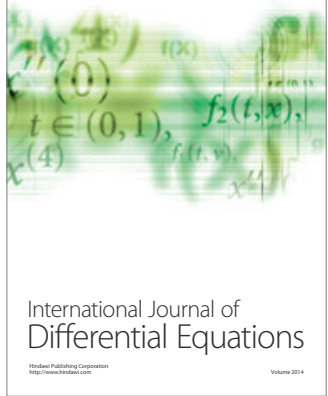
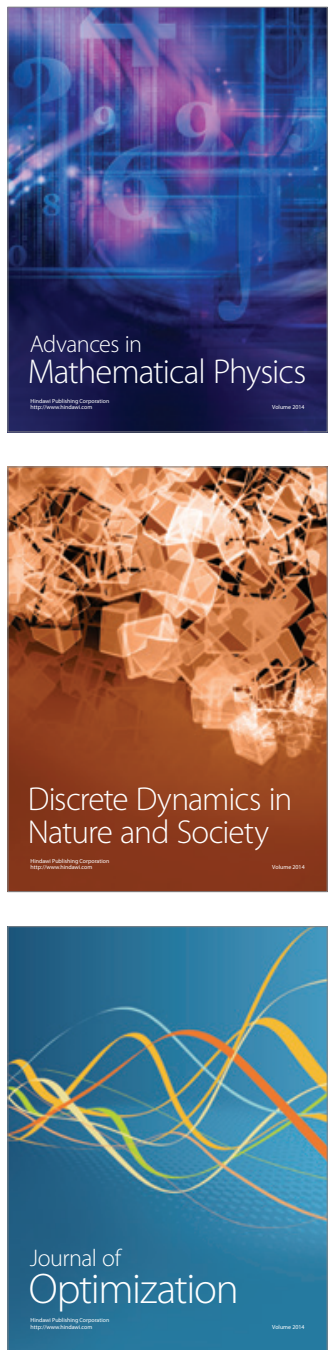